\title{
Existence Results of Initial Value Problems for Hybrid Fractional Sum-Difference Equations
}

\author{
Saowaluck Chasreechai ${ }^{1}$ and Thanin Sitthiwirattham ${ }^{2}{ }^{2}$ \\ ${ }^{1}$ Department of Mathematics, Faculty of Applied Science, King Mongkut's University of Technology North Bangkok, \\ Bangkok, Thailand \\ ${ }^{2}$ Mathematics Department, Faculty of Science and Technology, Suan Dusit University, Bangkok, Thailand
}

Correspondence should be addressed to Thanin Sitthiwirattham; thanin_sit@dusit.ac.th

Received 25 September 2017; Revised 3 December 2017; Accepted 2 January 2018; Published 30 January 2018

Academic Editor: Jorge E. Macías-Díaz

Copyright (c) 2018 Saowaluck Chasreechai and Thanin Sitthiwirattham. This is an open access article distributed under the Creative Commons Attribution License, which permits unrestricted use, distribution, and reproduction in any medium, provided the original work is properly cited.

We consider a hybrid fractional sum-difference initial value problem and a hybrid fractional sequential sum-difference initial value problem. The existence results of these two problems are proved by using the hybrid fixed point theorem for three operators in a Banach algebra and the generalized Krasnoselskii's fixed point theorem, respectively.

\section{Introduction}

As recognized that fractional difference calculus is a powerful tool used to describe many real world phenomena problems such as physics, chemistry, mechanics, control systems, flow in porous media, and electrical networks $[1,2]$, this is an impact on a researcher's motivation to develop the research works in this area. Basic definitions and properties of fractional difference calculus were proposed by Goodrich and Peterson [3]. The developments of the theory related to discrete fractional boundary value problems were studied by many authors (see [4-48]). In particular Sitthiwirattham [41, 42] studied three-point Caputo fractional difference-fractional sum boundary value problem for sequential Caputo fractional difference equation of the form

$$
\begin{aligned}
& \Delta_{C}^{\alpha}\left[\phi_{p}\left(\Delta_{C}^{\beta} x\right)\right](t) \\
& \quad=f(t+\alpha+\beta-1, x(t+\alpha+\beta-1)), \\
& \Delta_{C}^{\beta} x(\alpha-1)=0, \\
& x(\alpha+\beta+T)=\rho \Delta^{-\gamma} x(\eta+\gamma),
\end{aligned}
$$

and three-point fractional sum boundary value problem for sequential Riemann-Liouville fractional difference equation of the form

$$
\begin{aligned}
& \Delta_{\alpha}^{\alpha}\left(\Delta_{\alpha+\beta-1}^{\beta}+\lambda E_{\beta}\right) x(t) \\
& \quad=f(t+\alpha+\beta-1, x(t+\alpha+\beta-1)), \\
& x(\alpha+\beta-2)=0, \\
& x(\alpha+\beta+T)=\rho \Delta_{\alpha+\beta-1}^{-\gamma} x(\eta+\gamma),
\end{aligned}
$$

where $t \in \mathbb{N}_{0, T}, 0<\alpha, \beta \leq 1,1<\alpha+\beta \leq 2,0<\gamma \leq 1$, $\eta \in \mathbb{N}_{\alpha+\beta-1, \alpha+\beta+T-1}, \rho$ is a constant, $f: \mathbb{N}_{\alpha+\beta-2, \alpha+\beta+T} \times \mathbb{R} \rightarrow \mathbb{R}$ is a continuous function, $E_{\beta} x(t)=x(t+\beta-1)$, and $\phi_{p}$ is the $p$-Laplacian operator.

Calculus which deals with derivatives and integrals of arbitrary orders is known as hybrid differential equations (i.e., quadratic perturbation of a nonlinear differential equation). Hybrid fractional differential equations are initialized to be used to model successfully several physical phenomena (see [49-51]). Apparently, this issue has found numerous miscellaneous applications connected with real world problems as they appear in many fields of engineering and science, 
including biology, chemistry, diffusion, control theory, electromagnetic theory, fluid flow, signal and image processing, fractals theory, fitting of experimental data, potential theory, and viscoelasticity. For some recent developments on the topic, see [52-57]. Recently, there are several research works related to boundary value problems for hybrid differential equations (see [58-65]). For example, Sun et al. [60] studied the existence of solutions for the boundary value problem of fractional hybrid differential equations

$$
\begin{aligned}
D^{\nu}\left[\frac{x(t)}{f(t, x(t))}\right]+g(t, x(t)) & =0, \quad 0<t<1, \\
x(0) & =x(1)=0,
\end{aligned}
$$

where $D^{v}$ denotes the Riemann-Liouville fractional derivative of order $v, 1<v \leq 2$.

Sitho et al. [64] studied existence results for the following initial value problem for hybrid fractional integrodifferential equations,

$$
\begin{aligned}
D^{\alpha}\left[\frac{x(t)-\sum_{i=1}^{m} I^{\beta_{i}} h_{i}(t, x(t))}{f(t, x(t))}\right] & =F(t, x(t)), \\
t \in J:[0, T], & t(0)=0,
\end{aligned}
$$

and the initial value problem for hybrid fractional sequential integrodifferential equations,

$$
\begin{aligned}
& D^{\alpha}\left[\frac{D^{\omega} x(t)-\sum_{i=1}^{m} I^{\beta_{i}} h_{i}(t, x(t))}{f(t, x(t))}\right] \\
& \quad=G\left(t, x(t), I^{\gamma} x(t)\right), \quad t \in J, \\
& x(0)=D^{\omega} x(0)=0,
\end{aligned}
$$

where $D^{\alpha}$ denotes the Riemann-Liouville fractional derivative of order $\alpha, I^{\phi}$ is the Riemann-Liouville fractional integral of order $\phi>0, \phi \in\left\{\beta_{1}, \beta_{2}, \ldots, \beta_{m}\right\}, 0<\alpha, \omega \leq 1,1<\alpha+\omega \leq$ 2, and functions are $F \in C(J \times \mathbb{R}, \mathbb{R}), G \in C\left(J \times \mathbb{R}^{2}\right), \mathbb{R}$, $f \in C(J \times \mathbb{R}, \mathbb{R}-\{0\})$, and $h_{i} \in C(J \times \mathbb{R}, \mathbb{R})$ with $h_{i}(0,0)=0$, $i=1,2, \ldots, m$.

While the boundary value problem for hybrid fractional difference equations has not been studied, to fill this gap, we study a hybrid fractional difference initial value problem of the form,

$$
\begin{aligned}
& \Delta^{\alpha}\left[\frac{u(t)-\Delta^{-\gamma} p(t+\gamma, u(t+\gamma))}{f(t, u(t))}\right] \\
& \quad=F[t+\alpha, u(t+\alpha)], \\
& u(\alpha-1)=0,
\end{aligned}
$$

and a hybrid fractional sequential sum-difference initial value problem of the form

$$
\begin{aligned}
& \Delta^{\alpha}\left[\frac{\Delta^{\beta} u(t)-\Delta^{-\gamma} g(t+\beta+\gamma-1, u(t+\beta+\gamma-1))}{h(t, u(t))}\right] \\
& \quad=H[t+\alpha+\beta \\
& \left.\quad-1, u(t+\alpha+\beta-1), \Delta^{-\omega} u(t+\alpha+\beta+\omega-1)\right], \\
& u(\alpha+\beta-2)=\Delta^{\beta} u(\alpha-1)=0,
\end{aligned}
$$

where $t \in \mathbb{N}_{0, T}:=\{0,1, \ldots, T\}, \alpha, \beta, \gamma, \omega \in(0,1], 1<\alpha+$ $\beta \leq 2$ are given constants, $f \in C\left(\mathbb{N}_{\alpha-1, T+\alpha} \times \mathbb{R}, \mathbb{R}-\{0\}\right)$, $h \in C\left(\mathbb{N}_{\alpha+\beta-2, T+\alpha+\beta} \times \mathbb{R}, \mathbb{R}-\{0\}\right), p \in C\left(\mathbb{N}_{\alpha-1, T+\alpha} \times \mathbb{R}, \mathbb{R}\right)$ with $p(\alpha-1,0)=0, g \in C\left(\mathbb{N}_{\alpha+\beta-2, T+\alpha+\beta} \times \mathbb{R}, \mathbb{R}\right)$ with $g(\alpha+\beta-$ $2,0)=0, F \in C\left(\mathbb{N}_{\alpha-1, T+\alpha} \times \mathbb{R}, \mathbb{R}\right)$, and $H \in C\left(\mathbb{N}_{\alpha+\beta-2, T+\alpha+\beta} \times\right.$ $\mathbb{R} \times \mathbb{R}, \mathbb{R})$.

The article is organized as follows. In Section 2, we recall some definitions and basic lemmas used in this work. Then, we present the solutions of (6) and (7) by converting the problem to an equivalent summation equation. In Sections 3 and 4, we prove existence results of problems (6) and (7) by employing the hybrid fixed point theorem for three operators in a Banach algebra and the generalized Krasnoselskii's fixed point theorem, respectively. We end with some examples to illustrate our results in the last section.

\section{Preliminaries}

In what follows are the notations, definitions, and lemmas which are used in the main results.

Definition 1 (see [6]). Define the generalized falling function by $t^{\underline{\alpha}}:=\Gamma(t+1) / \Gamma(t+1-\alpha)$, for any $t$ and $\alpha$ for which the right-hand side is defined. If $t+1-\alpha$ is a pole of the Gamma function and $t+1$ is not a pole, then $t^{\underline{\alpha}}=0$.

Lemma 2 (see [4]). Assume that the factorial functions are well defined. If $t \leq r$, then $t^{\underline{\alpha}} \leq r^{\underline{\alpha}}$ for any $\alpha>0$.

Definition 3 (see [6]). For $\alpha>0$ and $f$ defined on $\mathbb{N}_{a}:=\{a, a+$ $1, \ldots\}$, the $\alpha$-order fractional sum of $f$ is defined by

$$
\Delta^{-\alpha} f(t):=\frac{1}{\Gamma(\alpha)} \sum_{s=a}^{t-\alpha}(t-\sigma(s))^{\frac{\alpha-1}{}} f(s),
$$

where $t \in \mathbb{N}_{a+\alpha}$ and $\sigma(s)=s+1$.

Definition 4 (see [6]). For $\alpha>0$ and $f$ defined on $\mathbb{N}_{a}$, the $\alpha$ order Riemann-Liouville fractional difference of $f$ is defined by

$$
\begin{aligned}
\Delta^{\alpha} f(t) & :=\Delta^{N} \Delta^{-(N-\alpha)} f(t) \\
& =\frac{1}{\Gamma(-\alpha)} \sum_{s=a}^{t+\alpha}(t-\sigma(s)) \frac{-\alpha-1}{-} f(s),
\end{aligned}
$$

where $t \in \mathbb{N}_{a+N-\alpha}$ and $N \in \mathbb{N}$ is satisfied with $0 \leq N-1<$ $\alpha<N$. 
Lemma 5 (see [5]). For any real number $v$ and any positive integer $p$, the following equality holds:

$$
\begin{aligned}
\Delta^{-\nu} \Delta^{p} f(t)= & \Delta^{p} \Delta^{-\nu} f(t) \\
& -\sum_{k=0}^{p-1} \frac{(t-a)^{\nu-p+k}}{\Gamma(\nu-p+k+1)} \Delta^{k} f(a),
\end{aligned}
$$

or $f$ is defined on $\mathbb{N}_{a}$.

Lemma 6 (see [4]). Let $0 \leq N-1<\alpha \leq N$. Then,

$$
\Delta^{-\alpha} \Delta^{\alpha} y(t)=y(t)+C_{1} t^{\frac{\alpha-1}{2}}+C_{2} t^{\frac{\alpha-2}{2}}+\cdots+C_{N} t^{\frac{\alpha-N}{}},
$$

for some $C_{i} \in \mathbb{R}$, with $1 \leq i \leq N$.

We provide the following lemma dealing with linear variant of the boundary value problems (6) and (7) and give a representation of the solution.

Lemma 7. Let $\alpha, \gamma \in(0,1], p \in C\left(\mathbb{N}_{\alpha-1, T+\alpha} \times \mathbb{R}, \mathbb{R}\right)$ with $p(\alpha-$ $1,0)=0, y \in C\left(\mathbb{N}_{\alpha-1, T+\alpha}, \mathbb{R}-\{0\}\right)$, and $h \in C\left(\mathbb{N}_{\alpha-1, T+\alpha}, \mathbb{R}\right)$. Then, for $t \in \mathbb{N}_{0, T}$, the problem

$$
\begin{aligned}
\Delta^{\alpha}\left[\frac{u(t)-\Delta^{-\gamma} p(t+\gamma, u(t+\gamma))}{y(t)}\right] & =h(t+\alpha), \\
u(\alpha-1) & =0,
\end{aligned}
$$

has the unique solution

$u(t)$

$$
\begin{aligned}
= & \frac{y(t)}{\Gamma(\alpha)} \sum_{s=0}^{t-\alpha}(t-\sigma(s))^{\frac{\alpha-1}{}} h(s+\alpha) \\
& +\frac{1}{\Gamma(\gamma)} \sum_{s=\alpha-\gamma-1}^{t-\gamma}(t-\sigma(s)) \frac{\gamma-1}{-} p(s+\gamma, u(s+\gamma)),
\end{aligned}
$$

for $t \in \mathbb{N}_{\alpha-1, T+\alpha}$.

Proof. Using the fractional sum of order $\alpha: \Delta^{-\alpha}$ for (12) and Lemmas 5 and 6, we obtain

$$
\begin{gathered}
\Delta^{-\alpha} h(t+\alpha)=\left[\frac{u(t)-\Delta^{-\gamma} p(t+\gamma, u(t+\gamma))}{y(t)}\right]-\frac{t^{\frac{\alpha-1}{}}}{\Gamma(\alpha)} \\
\cdot \Delta^{-(1-\alpha)}\left[\frac{u(\alpha-1)-\Delta^{-\gamma} p(\alpha-1+\gamma, u(\alpha-1+\gamma))}{y(\alpha-1)}\right] \\
=\left[\frac{u(t)-\Delta^{-\gamma} p(t+\gamma, u(t+\gamma))}{y(t)}\right]-\frac{t^{\alpha-1}}{\Gamma(\alpha)}
\end{gathered}
$$

$$
\begin{aligned}
& \cdot \Delta^{-(1-\alpha)}\left\{\frac{1}{y(\alpha-1)} \times[u(\alpha-1)\right. \\
& \left.\left.-\frac{1}{\Gamma(\gamma)} \sum_{s=\alpha-1}^{(\alpha+\gamma-1)-\gamma}(\alpha+\gamma-1-\sigma(s)) \frac{\gamma-1}{-} p(s, u(s))\right]\right\} \\
& =\left[\frac{u(t)-\Delta^{-\gamma} p(t+\gamma, u(t+\gamma))}{y(t)}\right]-\frac{t^{\frac{\alpha-1}{}}}{\Gamma(\alpha)} \\
& \cdot \Delta^{-(1-\alpha)}\left\{\frac{1}{y(\alpha-1)}[u(\alpha-1)\right. \\
& -\gamma p(\alpha-1, u(\alpha-1))]\} \text {, }
\end{aligned}
$$

for $t \in \mathbb{N}_{\alpha-1, T+\alpha}$.

Since $u(\alpha-1)=0, p(\alpha-1,0)=0$, and $y(\alpha-1) \neq 0$, it follows that

$$
\left[\frac{u(t)-\Delta^{-\gamma} p(t+\gamma, u(t+\gamma))}{y(t)}\right]=\Delta^{-\alpha} h(t+\alpha) .
$$

Thus, (13) holds. The proof is completed.

Lemma 8. Let $\alpha, \beta, \gamma \in(0,1], 1<\alpha+\beta \leq 2, g \in$ $C\left(\mathbb{N}_{\alpha+\beta-2, T+\alpha+\beta} \times \mathbb{R}, \mathbb{R}\right)$ with $g(\alpha+\beta-2,0)=0$, $x \in$ $C\left(\mathbb{N}_{\alpha+\beta-2, T+\alpha+\beta}, \mathbb{R}-\{0\}\right)$, and $k \in C\left(\mathbb{N}_{\alpha+\beta-2, T+\alpha+\beta}, \mathbb{R}\right)$ be given. Then, for $t \in \mathbb{N}_{0, T}$, the problem

$$
\begin{aligned}
& \Delta^{\alpha}\left[\frac{\Delta^{\beta} u(t)-\Delta^{-\gamma} g(t+\beta+\gamma-1, u(t+\beta+\gamma-1))}{x(t)}\right] \\
& \quad=k(t+\alpha+\beta-1), \\
& u(\alpha+\beta-2)=\Delta^{\beta} u(\alpha-1)=0,
\end{aligned}
$$

has the unique solution

$$
\begin{aligned}
& u(t)=\frac{1}{\Gamma(\alpha) \Gamma(\beta)} \sum_{s=\alpha-1}^{t-\beta}\left[(t-\sigma(s)) \frac{\beta-1}{x} x(s+\beta)\right. \\
& \left.\cdot \sum_{\xi=0}^{s-\alpha}(s-\sigma(\xi))^{\frac{\alpha-1}{2}} k(\xi+\alpha+\beta-1)\right]+\frac{1}{\Gamma(\gamma)} \\
& \cdot \sum_{s=\alpha+\beta-\gamma-2}^{t-\gamma}(t-\sigma(s)) \frac{\gamma-1}{g} g(s+\beta+\gamma, u(s+\beta+\gamma)),
\end{aligned}
$$


Proof. Using the fractional sum of order $\alpha: \Delta^{-\alpha}$ for (16) and Lemmas 5 and 6, we obtain

$$
\begin{aligned}
\Delta^{-\alpha} k & (t+\alpha+\beta-1) \\
= & {\left[\frac{\Delta^{\beta} u(t)-\Delta^{-\gamma} g(t+\beta+\gamma-1, u(t+\beta+\gamma-1))}{x(t)}\right] } \\
& -\frac{t^{\frac{\alpha-1}{}}}{\Gamma(\alpha)} \Delta^{-(1-\alpha)}\left[\frac{\Delta^{\beta} u(\alpha-1)-\Delta^{-\gamma} g(\alpha-1+\beta+\gamma-1, u(\alpha-1+\beta+\gamma-1))}{x(\alpha-1)}\right] \\
= & {\left.\left.\left[\frac{\Delta^{\beta} u(t)-\Delta^{-\gamma} g(t+\beta+\gamma-1, u(t+\beta+\gamma-1))}{x(t)}\right] \sum_{s=\alpha+\beta-2}(\alpha+\beta+\gamma-2-\sigma(s)) \frac{\gamma-1}{(1)} g(s, u(s))\right]\right\} } \\
& \left.\left.-\frac{t^{\frac{\alpha-1}{\Gamma(\alpha)}} \Delta^{-(1-\alpha)}\left\{\frac{1}{x(\alpha-1)} \times\left[\Delta^{\beta} u(\alpha-1)-\frac{1}{\Gamma(\gamma)} \sum_{(\alpha+\beta-2)-\gamma}\right.\right.}{\Gamma^{\beta}}\right]\right\} \\
= & -\frac{\Delta^{\beta} u(t)-\Delta^{-\gamma} g(t+\beta+\gamma-1, u(t+\beta+\gamma-1))}{\Gamma(\alpha)} \Delta^{-(1-\alpha)}\left\{\frac{1}{x(\alpha-1)}\left[\Delta^{\beta} u(\alpha-1)-\gamma g(\alpha+\beta-2, u(\alpha+\beta-2))\right]\right\},
\end{aligned}
$$

for $t \in \mathbb{N}_{\alpha-1, T+\alpha}$.

Since $u(\alpha+\beta-2)=\Delta^{\beta} u(\alpha-1)=0, g(\alpha+\beta-2,0)=0$, and $x(\alpha-1) \neq 0$, we have

$$
\begin{aligned}
& {\left[\frac{\Delta^{\beta} u(t)-\Delta^{-\gamma} g(t+\beta+\gamma-1, u(t+\beta+\gamma-1))}{x(t)}\right]} \\
& \quad=\Delta^{-\alpha} k(t+\alpha+\beta-1) .
\end{aligned}
$$

That can be arranged in the form

$$
\begin{aligned}
\Delta^{\beta} u(t)= & x(t) \Delta^{-\alpha} k(t+\alpha+\beta-1) \\
& +\Delta^{-\gamma} g(t+\beta+\gamma-1, u(t+\beta+\gamma-1)) .
\end{aligned}
$$

Using the fractional sum of order $\beta$ for (20), we obtain

$$
\begin{aligned}
u(t)= & \Delta^{-\beta}\left[x(t) \Delta^{-\alpha} k(t+\alpha+\beta-1)\right] \\
& +\Delta^{-\beta-\gamma} g(t+\beta+\gamma-1, u(t+\beta+\gamma-1)),
\end{aligned}
$$

for $t \in \mathbb{N}_{\alpha+\beta-2, T+\alpha+\beta}$.

Thus (17) holds. Our proof is completed.

Lemma 9 (Arzelá-Ascoli theorem [66]). A set of functions in $C[a, b]$ with the sup norm is relatively compact if and only if it is uniformly bounded and equicontinuous on $[a, b]$.

Lemma 10 (see [66]). If a set is closed and relatively compact, then it is compact.

\section{Hybrid Fractional Sum-Difference Initial Value Problem (6)}

In this section, we aim to show the existence results for problem (6). To accomplish this, we let $E=C\left(\mathbb{N}_{\alpha-1, T+\alpha}, \mathbb{R}\right)$ be a space of all functions $u$ and defined a norm and a multiplication in $E$ by

$$
\|u\|=\max _{t \in \mathbb{N}_{\alpha-1, T+\alpha}}|u(t)|,
$$

$$
(u v)(t)=u(t) v(t)
$$

In addition, we define operator $\mathscr{F}: E \rightarrow E$ by

$(\mathscr{F} u)(t)$

$$
\begin{aligned}
= & \frac{f(t, u(t))}{\Gamma(\alpha)} \sum_{s=0}^{t-\alpha}(t-\sigma(s)) \frac{\alpha-1}{} F((s+\alpha), u(s+\alpha)) \\
& +\frac{1}{\Gamma(\gamma)} \sum_{s=\alpha-\gamma-1}^{t-\gamma}(t-\sigma(s)) \frac{\gamma-1}{} p(s+\gamma, u(s+\gamma)) .
\end{aligned}
$$

Clearly, problem (6) has solutions if and only if operator $\mathscr{F}$ has fixed points. The first shows the existence and uniqueness of a solution to problem (6) by using the Banach contraction principle.

Theorem 11. Assume that $f \in C\left(\mathbb{N}_{\alpha-1, T+\alpha} \times \mathbb{R}, \mathbb{R}-\{0\}\right)$, $p \in C\left(\mathbb{N}_{\alpha-1, T+\alpha} \times \mathbb{R}, \mathbb{R}\right)$ with $p(\alpha-1,0)=0$, and $H \in$ $C\left(\mathbb{N}_{\alpha+\beta-2, T+\alpha+\beta} \times \mathbb{R} \times \mathbb{R}, \mathbb{R}\right)$. In addition, suppose that 
$\left(A_{1}\right)$ there exist constants $L_{1}, L_{2}>0$ such that for each $t \epsilon$ $\mathbb{N}_{\alpha-1, T+\alpha}$ and $u, v \in \mathbb{R}$

$$
\begin{aligned}
& |F(t, u)-F(t, v)| \leq L_{1}|u-v|, \\
& |p(t, u)-p(t, v)| \leq L_{2}|u-v|,
\end{aligned}
$$

$\left(A_{2}\right)$ there exists a positive function $\theta$ with bound $\|\theta\|$ such that for each $t \in \mathbb{N}_{\alpha-1, T+\alpha}$ and $u, v \in \mathbb{R}$

$$
|f(t, u)-f(t, v)| \leq \theta(t) .
$$

If $L_{1}\|\theta\|\left(T^{\underline{\alpha}} / \Gamma(\alpha+1)\right)+L_{2}\left((T+\alpha)^{\underline{\gamma}} / \Gamma(\gamma+1)\right)<1$, then problem (6) has a unique solution.

Proof. We shall show that $\mathscr{F}$ is a contraction. For any $u, v \in \mathscr{C}$ and for each $t \in \mathbb{N}_{\alpha-1, T+\alpha}$, we have

$$
\begin{aligned}
& |(\mathscr{F} u)(t)-(\mathscr{F} v)(t)| \leq \frac{\|f\|}{\Gamma(\alpha)} \sum_{s=0}^{t-\alpha}(t-\sigma(s))^{\frac{\alpha-1}{}} \\
& \cdot|F((s+\alpha), u(s+\alpha))-F((s+\alpha), v(s+\alpha))| \\
& +\frac{1}{\Gamma(\gamma)} \sum_{s=\alpha-\gamma-1}^{t-\gamma}(t-\sigma(s))^{\frac{\gamma-1}{}} \\
& \cdot|p(s+\gamma, u(s+\gamma))-p(s+\gamma, v(s+\gamma))| \\
& \leq \frac{\|\theta\|}{\Gamma(\alpha)} L_{1}\|u-v\| \sum_{s=0}^{T}(T+\alpha-\sigma(s))^{\frac{\alpha-1}{}}+\frac{1}{\Gamma(\gamma)} \\
& \cdot L_{2}\|u-v\| \sum_{s=\alpha-\gamma-1}^{T+\alpha-\gamma}(T+\alpha-\sigma(s))^{\frac{\gamma-1}{}} \leq \| u \\
& -v \|\left\{L_{1}\|\theta\| \frac{T^{\underline{\alpha}}}{\Gamma(\alpha+1)}+L_{2} \frac{(T+\alpha) \underline{\underline{\gamma}}}{\Gamma(\gamma+1)}\right\} .
\end{aligned}
$$

Thus, we have $\|(\mathscr{F} u)-(\mathscr{F} v)\|<\|u-v\|$.

Consequently, $\mathscr{F}$ is a contraction. Therefore, by the Banach fixed point theorem, we get that $\mathscr{F}$ has a fixed point which is a unique solution of problem (6).

In the second result, we deduce the existence of at least one solution of the initial value problem (6) by using the hybrid fixed point theorem for three operators in a Banach algebra. Clearly, $E$ is a Banach algebra with respect to the above norm and multiplication in it.

Theorem 12 (hybrid fixed point theorem for three operators in a Banach algebra [67]). Let $S$ be a nonempty, closed convex, and bounded subset of the Banach algebra $E$, and let $A, C$ : $E \rightarrow E$ and $B: S \rightarrow E$ be three operators such that

(i) $A$ and $C$ are Lipschitzian with Lipschitz constants $\delta$ and $\rho$, respectively,

(ii) $B$ is completely continuous,

(iii) $x=A x B y+C x \Rightarrow x \in S$ for all $y \in S$,

(iv) $\delta M+\rho<1$, where $M=\|B(S)\|$.

Then, the operator equation $A x B x+C x=x$ has a solution.
Theorem 13. Assume that $f \in C\left(\mathbb{N}_{\alpha-1, T+\alpha} \times \mathbb{R}, \mathbb{R}-\{0\}\right)$, $p \in C\left(\mathbb{N}_{\alpha-1, T+\alpha} \times \mathbb{R}, \mathbb{R}\right)$ with $p(\alpha-1,0)=0$, and $H \in$ $C\left(\mathbb{N}_{\alpha+\beta-2, T+\alpha+\beta} \times \mathbb{R} \times \mathbb{R}, \mathbb{R}\right)$. In addition, suppose that

$\left(H_{1}\right)$ there exist two positive functions $\phi$ and $\psi$ with bound $\|\phi\|$ and $\|\psi\|$, respectively, such that for each $t \in$ $\mathbb{N}_{\alpha-1, T+\alpha}$ and $u, v \in \mathbb{R}$

$$
\begin{aligned}
& |f(t, u)-f(t, v)| \leq \phi(t)|u-v|, \\
& |p(t, u)-p(t, v)| \leq \psi(t)|u-v|,
\end{aligned}
$$

$\left(H_{2}\right)$ there exists a function $\omega \in C\left(\mathbb{N}_{\alpha-1, T+\alpha}, \mathbb{R}^{+}\right)$and a continuous nondecreasing function $\Psi:[0, \infty) \rightarrow$ $(0, \infty)$ such that for each $(t, u) \in \mathbb{N}_{\alpha-1, T+\alpha} \times \mathbb{R}$

$$
|F(t, u)| \leq \omega(t) \Psi(|u|),
$$

$\left(H_{3}\right)$ there exists a number

$R$

$$
\geq \frac{\widehat{f}_{0}\left(\|\omega\| \Psi(R) T^{\alpha} / \Gamma(\alpha+1)\right)+\widehat{p}_{0}\left((T+\alpha)^{\gamma} / \Gamma(\gamma+1)\right)}{1-\|\phi\|\left(\|\omega\| \Psi(R) T^{\alpha} / \Gamma(\alpha+1)\right)-\|\psi\|\left((T+\alpha)^{\gamma} / \Gamma(\gamma+1)\right)},
$$

where $\hat{f}_{0}=\max _{t \in \mathbb{N}_{\alpha-1, T+\alpha}}|f(t, 0)|, \quad \hat{p}_{0}=$ $\max _{t \in \mathbb{N}_{\alpha-1, T+\alpha}}|p(t, 0)|$, and

$$
\|\phi\| \frac{\|\omega\| \Psi(R) T^{\alpha}}{\Gamma(\alpha+1)}+\|\psi\| \frac{(T+\alpha)^{\gamma}}{\Gamma(\gamma+1)}<1 .
$$

Then problem (6) has a unique solution on $\mathbb{N}_{\alpha-1, T+\alpha}$.

Proof. Define subset $S$ of $E$ as

$$
S=\{u \in E:\|u\| \leq R\} .
$$

We see that $S$ is closed, convex, and bounded subset of the Banach space $\mathscr{C}$. By Lemma 7 , we define three operators $\mathscr{A}$ : $E \rightarrow E, \mathscr{B}: S \rightarrow E$, and $\mathscr{C}: E \rightarrow E$ by

$$
\begin{aligned}
& \mathscr{A} u(t):=f(t, u(t)), \quad t \in \mathbb{N}_{\alpha-1, T+\alpha}, \\
& \mathscr{B} u(t):=\frac{1}{\Gamma(\alpha)} \sum_{s=0}^{t-\alpha}(t-\sigma(s))^{\frac{\alpha-1}{w}} F(s+\alpha, u(s+\alpha)), \\
& t \in \mathbb{N}_{\alpha-1, T+\alpha},
\end{aligned}
$$

$\mathscr{C} u(t)$

$$
\begin{array}{r}
:=\frac{1}{\Gamma(\gamma)} \sum_{s=\alpha-\gamma-1}^{t-\gamma}(t-\sigma(s)) \frac{\gamma-1}{-} p(s+\gamma, u(s+\gamma)), \\
t \in \mathbb{N}_{\alpha-1, T+\alpha .}
\end{array}
$$

Note that problem (6) has solutions if and only if the operator $u=\mathscr{A} u \mathscr{B} v+\mathscr{C} u$ has fixed points.

To show that all operators satisfy all the conditions of Theorem 12, we proceed with the following steps.

Step 1. Prove that $\mathscr{A}$ and $\mathscr{C}$ are Lipschitzian on $E$. 
For any $u, v \in \mathbb{R}$ and for each $t \in \mathbb{N}_{\alpha-1, T+\alpha}$, then by $\left(H_{1}\right)$, we have

$$
\begin{aligned}
& |\mathscr{A} u(t)-\mathscr{A} v(t)|=|f(t, u(t))-f(t, v(t))| \leq \phi(t) \mid u \\
& -v \mid \leq\|\phi\|\|u-v\|, \\
& |\mathscr{C} u(t)-\mathscr{C} v(t)|=\frac{1}{\Gamma(\gamma)} \sum_{s=\alpha-\gamma-1}^{t-\gamma}(t-\sigma(s)) \frac{\gamma-1}{} \\
& \cdot|p(s+\gamma, u(s+\gamma))-p(s+\gamma, v(s+\gamma))| \leq \frac{1}{\Gamma(\gamma)} \\
& \cdot \sum_{s=\alpha-\gamma-1}^{T+\alpha-\gamma}(T+\alpha-\sigma(s)) \frac{\gamma-1}{-} \psi(s)|u-v| \\
& \leq \frac{\|\psi(s)\|(T+\alpha)^{\gamma}}{\Gamma(\gamma+1)}\|u-v\| .
\end{aligned}
$$

This implies that, for all $u, v \in \mathbb{R}$,

$$
\begin{aligned}
& \|\mathscr{A} u(t)-\mathscr{A} v(t)\| \leq\|\phi\|\|u-v\|, \\
& \|\mathscr{C} u(t)-\mathscr{C} v(t)\| \leq \frac{\|\psi(s)\|(T+\alpha)^{\gamma}}{\Gamma(\gamma+1)}\|u-v\| .
\end{aligned}
$$

Therefore, $\mathscr{A}$ and $\mathscr{C}$ are Lipschitzian on $E$ with Lipschitz constants $\|\phi\|$ and $\|\psi(s)\|(T+\alpha)^{\gamma} / \Gamma(\gamma+1)$.

Step 2 . We prove that $\mathscr{B}$ is completely continuous on $S$.

Since $F$ is continuous, the operator $\mathscr{B}$ is continuous on $S$. Next, we will prove that the set $\mathscr{B}(S)$ is uniformly bounded in $S$. For any $u \in S$, we find that

$$
\begin{aligned}
|\mathscr{B} u(t)| & \leq\left|\frac{1}{\Gamma(\alpha)} \sum_{s=0}^{t-\alpha}(t-\sigma(s))^{\alpha-1} \omega(s) \Psi(R)\right| \\
& \leq \frac{\|\omega\| \Psi(R) T^{\alpha}}{\Gamma(\alpha+1)}=: K,
\end{aligned}
$$

for all $t \in \mathbb{N}_{\alpha-1, T+\alpha}$. Therefore, $\|\mathscr{B}\| \leq K$, which shows that $\mathscr{B}$ is uniformly bounded on $S$.

Next we show that $\mathscr{B}(S)$ is an equicontinuous set in $E$. For any $\epsilon>0$, there exists a positive constant $\delta$ such that, for $t_{1}, t_{2} \in \mathbb{N}_{\alpha-1, T+\alpha}$

$$
\left|t_{2}^{\alpha}-t_{1}^{\alpha}\right|<\frac{\Gamma(\alpha+1)}{\|\omega\| \Psi(R)}, \quad \text { whenever }\left|t_{2}-t_{1}\right|<\delta .
$$

Then we obtain

$$
\begin{aligned}
& \left|(\mathscr{B} x)\left(t_{2}\right)-(\mathscr{B} x)\left(t_{1}\right)\right| \\
& \quad \leq \frac{\|\omega\| \Psi(R)}{\Gamma(\alpha)} \mid \sum_{s=0}^{t_{2}-\alpha}\left(t_{2}-\sigma(s)\right)^{\frac{\alpha-1}{}} \\
& \quad-\sum_{s=0}^{t_{1}-\alpha}\left(t_{1}-\sigma(s)\right)^{\frac{\alpha-1}{}}\left|\leq \frac{\|\omega\| \Psi(R)}{\Gamma(\alpha+1)}\right| t_{2}^{\alpha}-t_{1}^{\alpha} \mid<\epsilon .
\end{aligned}
$$

This implies that the set $\mathscr{B}(S)$ is an equicontinuous set. From the Arzelá-Ascoli theorem, we find that $\mathscr{B}$ is completely continuous.

Step 3. $u=\mathscr{A} u \mathscr{B} v+\mathscr{C} u \Rightarrow u \in S$ for all $v \in S$.
Let $u \in E$ and $v \in S$ be arbitrary elements such that $u=$ $\mathscr{A} u \mathscr{B} v+\mathscr{C} u$. Then,

$$
\begin{aligned}
& |u(t)| \leq|\mathscr{A} u(t)||\mathscr{B} v(t)|+|\mathscr{C} u(t)| \leq \frac{|f(t, u(t))|}{\Gamma(\alpha)} \\
& \cdot \sum_{s=0}^{t-\alpha}(t-\sigma(s))^{\frac{\alpha-1}{}}|F(s+\alpha, u(s+\alpha))|+\frac{1}{\Gamma(\gamma)} \\
& \cdot \sum_{s=\alpha-1}^{t}(t+\gamma-\sigma(s)) \frac{\gamma-1}{-}|p(s, u(s))| \\
& \leq \frac{|f(t, u(t))-f(t, 0)|+|f(t, 0)|}{\Gamma(\alpha)} \\
& \cdot \sum_{s=0}^{T}(T+\alpha-\sigma(s)) \frac{\alpha-1}{\|\omega\|}\left(\Psi(R)+\frac{1}{\Gamma(\gamma)}\right. \\
& \cdot \sum_{s=\alpha-1}^{T+\alpha}(T+\alpha+\gamma-\sigma(s)) \frac{\gamma-1}{} \\
& \cdot(|p(s, u(s))-p(t, 0)|+|p(t, 0)|) \leq(R\|\phi\| \\
& \left.+\widehat{f}_{0}\right) \frac{\|\omega\| \Psi(R) T^{\alpha}}{\Gamma(\alpha+1)}+\left(R\|\psi\|+\widehat{p}_{0}\right) \frac{(T+\alpha)^{\gamma}}{\Gamma(\gamma+1)} .
\end{aligned}
$$

We find that

$$
\begin{aligned}
\|u\| \leq & \left(R\|\phi\|+\widehat{f}_{0}\right) \frac{\|\omega\| \Psi(R) T^{\alpha}}{\Gamma(\alpha+1)} \\
& +\left(R\|\psi\|+\widehat{p}_{0}\right) \frac{(T+\alpha)^{\gamma}}{\Gamma(\gamma+1)} \leq R .
\end{aligned}
$$

Therefore, $u \in S$.

Step 4. We prove that $\delta M+\rho<1$. Since

$$
\begin{aligned}
M & =\|\mathscr{B}(S)\|=\sup _{u \in S}\left\{\max _{t \in \mathbb{N}_{\alpha-1, T+\alpha}}|\mathscr{B} u(t)|\right\} \\
& \leq \frac{\|\omega\| \Psi(R) T^{\alpha}}{\Gamma(\alpha+1)},
\end{aligned}
$$

and by $\left(H_{3}\right)$, we have

$$
\|\phi\| M+\|\psi\| \frac{(T+\alpha)^{\gamma}}{\Gamma(\gamma+1)}<1,
$$

with $\delta=\|\phi\|$ and $\rho=\|\psi\|\left((T+\alpha)^{\gamma} / \Gamma(\gamma+1)\right)$.

We see that all the conditions of Theorem 12 are satisfied. Hence, the operator equation $u=\mathscr{A} u \mathscr{B} v+\mathscr{C} u$ has a solution in $S$. In consequence, problem (6) has a solution on $\mathbb{N}_{\alpha-1, T+\alpha}$. This completes the proof.

\section{Hybrid Fractional Sequential Sum-Difference Initial Value Problem (7)}

In this section, we prove existence results of problem (7). To accomplish this, we denote that $X=C\left(\mathbb{N}_{\alpha+\beta-2, T+\alpha+\beta}, \mathbb{R}\right)$ and define the space of all functions $u$ with the norm by

$$
\|u\|=\max _{t \in \mathbb{N}_{\alpha+\beta-2, T+\alpha+\beta}}|u(t)| .
$$


In addition, we define the operator $\mathscr{H}: X \rightarrow X$ by

$$
\begin{aligned}
& (\mathscr{H} u)(t)=\frac{1}{\Gamma(\alpha) \Gamma(\beta)} \sum_{s=\alpha-1}^{t-\beta}\left[(t-\sigma(s)) \frac{\beta-1}{}\right. \\
& \cdot h((s+\beta), u(s+\beta)) \times \sum_{\xi=0}^{s-\alpha}(s-\sigma(\xi))^{\frac{\alpha-1}{}} \\
& \cdot H((\xi+\alpha+\beta-1), u(\xi+\alpha+\beta-1))]+\frac{1}{\Gamma(\gamma)} \\
& \cdot \sum_{s=\alpha+\beta-\gamma-2}^{t-\gamma}(t-\sigma(s)) \frac{\gamma-1}{g} g(s+\beta+\gamma, u(s+\beta+\gamma)) .
\end{aligned}
$$

Clearly, problem (7) has solutions if and only if the operator $\mathscr{H}$ has fixed points. The first shows the existence and uniqueness of a solution to problem (7) by using the Banach contraction principle.

Theorem 14. Assume that $h \in C\left(\mathbb{N}_{\alpha+\beta-2, T+\alpha+\beta} \times \mathbb{R}, \mathbb{R}-\{0\}\right)$, $g \in C\left(\mathbb{N}_{\alpha+\beta-2, T+\alpha+\beta} \times \mathbb{R}, \mathbb{R}\right)$ with $g(\alpha+\beta-2,0)=0$, and $H \in C\left(\mathbb{N}_{\alpha+\beta-2, T+\alpha+\beta} \times \mathbb{R} \times \mathbb{R}, \mathbb{R}\right)$. In addition, suppose that
$\left(B_{1}\right)$ there exist constants $M_{1}, M_{2}, N>0$ such that for each $t \in \mathbb{N}_{\alpha+\beta-2, T+\alpha+\beta}$ and $u, v, u^{*}, v^{*} \in \mathbb{R}$

$$
\begin{aligned}
& \left|H\left(t, u, u^{*}\right)-H\left(t, v, v^{*}\right)\right| \\
& \quad \leq M_{1}|u-v|+M_{2}\left|u^{*}-v^{*}\right|, \\
& |g(t, u)-g(t, v)| \leq N|u-v|,
\end{aligned}
$$

$\left(B_{2}\right)$ there exists a positive function $\vartheta$ with bound $\|\vartheta\|$ such that for each $t \in \mathbb{N}_{\alpha+\beta-2, T+\alpha+\beta}$ and $u, v \in \mathbb{R}$

$$
|h(t, u)-h(t, v)| \leq \vartheta(t) .
$$

If $\Omega<1$ then problem (7) has a unique solution, where

$$
\begin{aligned}
\Omega:= & \frac{\|\vartheta\|}{\Gamma(\alpha) \Gamma(\beta)}(T+\beta) \frac{\beta-1}{}(T+\alpha+\beta)^{\frac{\alpha-1}{}} \\
& \cdot\left[M_{1}+M_{2} \frac{(T+\alpha+\beta)^{\underline{\omega}}}{\Gamma(\omega+1)}\right]+\frac{N(T+\alpha+\beta)^{\underline{\gamma}}}{\Gamma(\gamma+1)} .
\end{aligned}
$$

Proof. We shall show that $\mathscr{H}$ is a contraction. For any $u, v \in \mathscr{C}$ and for each $t \in \mathbb{N}_{\alpha-1, T+\alpha}$, we have

$$
\begin{aligned}
& |(\mathscr{H} u)(t)-(\mathscr{H} v)(t)| \leq \frac{\|h\|}{\Gamma(\alpha) \Gamma(\beta)} \sum_{s=\alpha-1}^{t-\beta}\left[(t-\sigma(s)) \frac{\beta-1}{}\right. \\
& \left.\cdot \sum_{\xi=0}^{s-\alpha}(s-\sigma(\xi))^{\frac{\alpha-1}{}}|H((\xi+\alpha+\beta-1), u(\xi+\alpha+\beta-1))-H((\xi+\alpha+\beta-1), v(\xi+\alpha+\beta-1))|\right]+\frac{1}{\Gamma(\gamma)} \\
& \cdot \sum_{s=\alpha+\beta-\gamma-2}^{t-\gamma}(t-\sigma(s)) \frac{\gamma-1}{\mid g}(s+\beta+\gamma, u(s+\beta+\gamma))-g(s+\beta+\gamma, v(s+\beta+\gamma)) \mid \leq \frac{\|\vartheta\|}{\Gamma(\alpha) \Gamma(\beta)}
\end{aligned}
$$

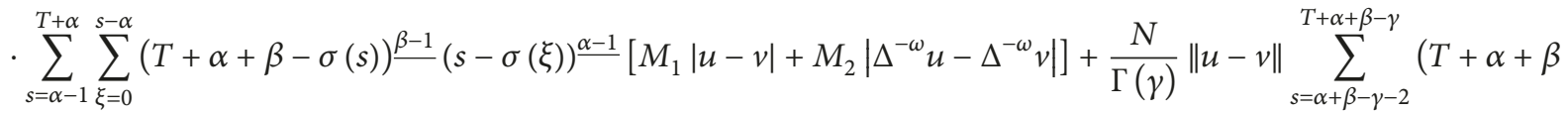

$$
\begin{aligned}
& -\sigma(s))^{\frac{\gamma-1}{}} \leq \frac{\|\vartheta\|}{\Gamma(\alpha) \Gamma(\beta)}\left[M_{1}+M_{2} \frac{(T+\alpha+\beta)^{\underline{\omega}}}{\Gamma(\omega+1)}\right]\|u-v\|(T+\beta)^{\frac{\beta-1}{}}(T+\alpha+\beta)^{\frac{\alpha-1}{}}+\frac{N}{\Gamma(\gamma)}\|u-v\|(T+\alpha+\beta)^{\underline{\gamma}}
\end{aligned}
$$

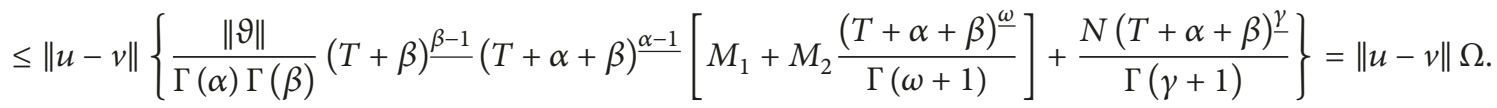

Thus, we have $\|(\mathscr{H} u)-(\mathscr{H} v)\|<\|u-v\|$.

Consequently, $\mathscr{H}$ is a contraction. Therefore, by the Banach fixed point theorem, we get that $\mathscr{H}$ has a fixed point which is a unique solution of problem (7).

In the second result, we deduce the existence of at least one solution of the initial value problem (7) by using the generalized Krasnoselskii’s fixed point theorem.

Theorem 15 (generalized Krasnoselskii's fixed point theorem [68]). Let $M$ be a nonempty, closed convex, and bounded subset of the Banach space $X$. Let $A: X \rightarrow X$ and $B: M \rightarrow X$ be two operators such that

(i) A is a contraction,

(ii) B is completely continuous,

(iii) $x=A x+B y$ for all $y \in M \Rightarrow x \in M$.

Then, the operator equation $A x+B x=x$ has a solution. 
Theorem 16. Assume that $h \in C\left(\mathbb{N}_{\alpha+\beta-2, T+\alpha+\beta} \times \mathbb{R}, \mathbb{R}-\{0\}\right)$, $g \in C\left(\mathbb{N}_{\alpha+\beta-2, T+\alpha+\beta} \times \mathbb{R}, \mathbb{R}\right)$ with $g(\alpha+\beta-2,0)=0$, and $H \in C\left(\mathbb{N}_{\alpha+\beta-2, T+\alpha+\beta} \times \mathbb{R} \times \mathbb{R}, \mathbb{R}\right)$. In addition, suppose that

$\left(K_{1}\right)$ there exist two positive functions $\vartheta$ and $\theta$ with bound $\|\vartheta\|$ and $\|\theta\|$, respectively, such that, for each $t \in$ $\mathbb{N}_{\alpha+\beta-2, T+\alpha+\beta}$ and $u, v, \Delta^{-\omega} u, \Delta^{-\omega} v \in \mathbb{R}$,

$$
\begin{aligned}
& |h(t, u)-h(t, v)| \leq \mathcal{V}(t)|u-v|, \\
& \left|H\left[t, u, \Delta^{-\omega} u\right]-H\left[t, u, \Delta^{-\omega} v\right]\right| \\
& \quad \leq \theta(t)\left(|u-v|-\left|\Delta^{-\omega} u-\Delta^{-\omega} v\right|\right),
\end{aligned}
$$

$\left(K_{2}\right)$ there exist functions $\mu, \nu, \kappa \in C\left(\mathbb{N}_{\alpha+\beta-2, T+\alpha+\beta}, \mathbb{R}^{+}\right)$ such that

$$
\begin{aligned}
&|h(t, u)| \leq \mu(t) \quad \forall(t, u) \in \mathbb{N}_{\alpha+\beta-2, T+\alpha+\beta} \times \mathbb{R}, \\
&\left|H\left(t, u, \Delta^{-\omega} u\right)\right| \leq \nu(t) \\
& \forall\left(t, u, \Delta^{-\omega} u\right) \in \mathbb{N}_{\alpha+\beta-2, T+\alpha+\beta} \times \mathbb{R} \times \mathbb{R}, \\
&|g(t, u)| \leq \kappa(t) \quad \forall(t, u) \in \mathbb{N}_{\alpha+\beta-2, T+\alpha+\beta} \times \mathbb{R} .
\end{aligned}
$$

If

$$
\begin{aligned}
& \frac{(T+\alpha+\beta)^{\beta}}{\Gamma(\beta+1)}\left\{\frac{\|\vartheta\|\|\nu\|(T+\alpha+\beta)^{\alpha}}{\Gamma(\alpha+1)}\right. \\
& \left.+\|\mu\|\|\theta\|\left(1+\frac{(T+\alpha+\beta)^{\omega}}{\Gamma(\omega+1)}\right)\right\}<1,
\end{aligned}
$$

then problem (7) has a unique solution on $\mathbb{N}_{\alpha+\beta-2, T+\alpha+\beta}$.

Proof. Let $\max _{t \in \mathbb{N}_{\alpha+\beta-2, T+\alpha+\beta}}|\mu(t)|=\quad\|\mu\|$, $\max _{t \in \mathbb{N}_{\alpha+\beta-2, T+\alpha+\beta}}|\nu(t)|=\|\nu\|$, and $\max _{t \in \mathbb{N}_{\alpha+\beta-2, T+\alpha+\beta}}|\kappa(t)|=\|\kappa\|$, and choose a constant

$$
R \geq \frac{\|\kappa\|(T+\alpha+\beta)^{\gamma}}{\Gamma(\gamma+1)}+\frac{\|\mu\|\|\nu\|(T+\alpha+\beta)^{\alpha+\beta}}{\Gamma(\alpha+1) \Gamma(\beta+1)} .
$$

We consider $B_{R}=\{u \in X:\|u\| \leq R\}$. Define four operators $\mathscr{P}: X \rightarrow X, \mathbb{Q}: B_{R} \rightarrow X$, and $\mathcal{S}: X \rightarrow X$ by

$$
\begin{aligned}
& \mathscr{P} u(t):=h(t, u(t)), \\
& \mathcal{Q} u(t):=\frac{1}{\Gamma(\alpha)} \sum_{s=0}^{t-\alpha}(t-\sigma(s))^{\alpha-1} \\
& \cdot H(s+\alpha+\beta-1, u(s+\alpha+\beta-1)), \\
& \mathcal{S u}(t):=\frac{1}{\Gamma(\gamma)} \sum_{s=\alpha+\beta-\gamma-2}^{t-\gamma}(t-\sigma(s)) \frac{\gamma-1}{} \\
& \cdot g(s+\beta+\gamma, u(s+\beta+\gamma)),
\end{aligned}
$$

for $t \in \mathbb{N}_{\alpha+\beta-2, T+\alpha+\beta}$, and

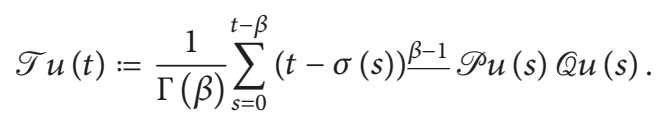

Problem (7) has solutions if and only if the operator $u=\delta \mathcal{S} u+$ $\mathscr{T} u$ has fixed points.

The proof is divided into three steps as follows.

Step 1 . Verify $u=\delta \mathcal{S}+\mathscr{T} u$ map bounded sets into bounded sets in $B_{R}$.

For each $v \in B_{R}$, we obtain

$$
\begin{aligned}
& |u(t)|=|\delta \mathcal{S u}(t)+\mathscr{T} u(t)| \leq \frac{1}{\Gamma(\gamma)} \\
& \cdot \sum_{s=\alpha+\beta-\gamma-2}^{t-\gamma}(t-\sigma(s)) \frac{\gamma-1}{\mid}|g(s+\beta+\gamma, u(s+\beta+\gamma))| \\
& +\frac{1}{\Gamma(\beta)} \sum_{s=0}^{t-\beta}(t-\sigma(s)) \frac{\beta-1}{\mid}|\mathscr{P} u(s)||Q \mathcal{Q} u(s)| \leq \frac{\|\kappa\|}{\Gamma(\gamma)} \\
& \cdot \sum_{s=\alpha+\beta-\gamma-2}^{T+\alpha+\beta-\gamma}(T+\alpha+\beta-\sigma(s))^{\gamma-1}+\frac{\|\mu\|\|\nu\|}{\Gamma(\alpha) \Gamma(\beta)} \\
& \cdot \sum_{s=0}^{T+\alpha}\left[(T+\alpha+\beta-\sigma(s))^{\beta-1} \sum_{\xi=0}^{s-\alpha}(\xi-\sigma(\xi))^{\alpha-1}\right] \\
& \leq \frac{\|\kappa\|(T+\alpha+\beta)^{\gamma}}{\Gamma(\gamma+1)}+\frac{\|\mu\|\|\nu\|(T+\alpha)^{\alpha+\beta}}{\Gamma(\alpha+1) \Gamma(\beta+1)} \leq R .
\end{aligned}
$$

Thus, $\|u\| \leq R$. This implies that $\delta u+\mathscr{T} u$ is uniformly bounded.

Hence, condition (iii) of Theorem 15 holds.

Step 2. Check that $\mathscr{T}$ is contraction mapping.

For any $u, v \in \mathbb{R}$ and for each $t \in \mathbb{N}_{\alpha+\beta-2, T+\alpha+\beta}$, by $\left(K_{1}\right)$, we have

$$
\begin{aligned}
& |\mathscr{T} u(t)-\mathscr{T} v(t)| \leq \frac{1}{\Gamma(\beta)} \mid \sum_{s=0}^{t-\beta}(t-\sigma(s)) \frac{\beta-1}{}[|\mathscr{P} u(s)| \\
& \left.\cdot|Q u(s)|-\left|\mathscr{P}_{v}(s)\right||Q \mathcal{Q} v(s)|\right]\left|=\frac{1}{\Gamma(\beta)}\right| \sum_{s=0}^{t-\beta}(t \\
& -\sigma(s)) \frac{\beta-1}{=}[|\mathscr{Q} u(s)|(|\mathscr{P} u(s)|-|\mathscr{P} v(s)|)+|\mathscr{P} v(s)| \\
& \cdot(|Q u(s)|-|Q \mathcal{Q} v(s)|)]\left|=\frac{1}{\Gamma(\beta)}\right| \sum_{s=0}^{t-\beta}\left(t-\sigma(s) \frac{\beta-1}{\underline{\beta}}\right. \\
& \left\{\frac{\|\vartheta\|\|\nu\|(T+\alpha+\beta)^{\alpha}}{\Gamma(\alpha+1)}\|u-v\|+\|\mu\|\|\theta\|\right. \\
& \left.\cdot\left(\|u-v\|+\|u-v\| \frac{1}{\Gamma(\omega)} \sum_{s=\alpha+\beta-2}^{t}(t-\sigma(s))^{\omega-1}\right)\right\} \mid \\
& \leq\|u-v\| \frac{(T+\alpha+\beta)^{\beta}}{\Gamma(\beta+1)}\left\{\frac{\|\vartheta\|\|\nu\|(T+\alpha+\beta)^{\alpha}}{\Gamma(\alpha+1)}\right. \\
& \left.+\|\mu\|\|\theta\|\left(1+\frac{(T+\alpha+\beta)^{\omega}}{\Gamma(\omega+1)}\right)\right\} .
\end{aligned}
$$


By (50), $\mathscr{T}$ is a contraction mapping. Hence, condition (i) of Theorem 15 holds.

Step 3. Check that $\mathcal{S}$ is completely continuous on $B_{R}$.

The operator $\mathcal{S}$ is obviously continuous on $B_{R}$. Furthermore, $\mathcal{S}$ is uniformly bounded on $B_{R}$ since

$$
\|\delta\| \leq \frac{\|\kappa\|(T+\alpha+\beta)^{\gamma}}{\Gamma(\gamma+1)} .
$$

For any $\epsilon>0$, there exists a positive constant $\delta$ such that for $t_{1}, t_{2} \in \mathbb{N}_{\alpha+\alpha+\beta, T+\alpha+\beta}$

$$
\left|t_{2}^{\gamma}-t_{1}^{\gamma}\right|<\frac{\Gamma(\gamma+1)}{\|\kappa\|}, \quad \text { whenever }\left|t_{2}-t_{1}\right|<\delta \text {. }
$$

Then,

$$
\begin{aligned}
& \left|(\mathcal{S} x)\left(t_{2}\right)-(\mathcal{S} x)\left(t_{1}\right)\right| \\
& \leq \frac{1}{\Gamma(\gamma)} \mid \sum_{s=\alpha+\beta-\gamma-2}^{t_{2}-\gamma}\left(t_{2}-\sigma(s)\right) \frac{\gamma-1}{} \\
& \cdot|g(s+\beta+\gamma, u(s+\beta+\gamma))| \\
& -\sum_{s=\alpha+\beta-\gamma-2}^{t_{1}-\gamma}\left(t_{1}-\sigma(s)\right) \frac{\gamma-1}{} \\
& \cdot|g(s+\beta+\gamma, u(s+\beta+\gamma))| \leq \frac{\|\kappa\|}{\Gamma(\gamma+1)} \mid t_{2}^{\gamma} \\
& -t_{1}^{\gamma} \mid<\epsilon .
\end{aligned}
$$

This implies that the set $\mathcal{S}\left(B_{R}\right)$ is an equicontinuous set. Therefore, by the Arzelá-Ascoli theorem, we find that $\mathcal{S}$ is completely continuous. Hence, condition (ii) of Theorem 15 holds.

We see that all the assumptions of Theorem 15 are satisfied. Therefore, we can conclude that problem (7) has at least one solution. The proof is completed.

\section{Examples}

In this section, we provide some examples to illustrate our results.

Example 1. Consider the following fractional difference initial value problem:

$$
\begin{aligned}
& \Delta^{1 / 2}\left[\frac{u(t)-\Delta^{-1 / 3} p(t+1 / 3, u(t+1 / 3))}{f(t, u(t))}\right] \\
& =F\left[t+\frac{1}{2}, u\left(t+\frac{1}{2}\right)\right], \quad t \in \mathbb{N}_{0,10}, \\
& u\left(-\frac{1}{2}\right)=0,
\end{aligned}
$$

where

$$
\begin{aligned}
& p(t, u(t))=\frac{u^{2}(t)+4|u(t)|}{3+|u(t)|} \cdot \frac{t \sin ^{2} 2 \pi t}{5000\left(2+e^{-t}\right)}, \\
& f(t, u(t))=\frac{10\left(2 \cos ^{2} 2 \pi t+3 t\right)}{3(100 \pi-2 t)^{2}} \cdot \frac{u^{2}(t)+3|u(t)|}{|u(t)|+10}, \\
& F[t, u(t)]=\frac{\left(t^{2}+5\right)(8|u(t)|+10 \pi)}{80(100 \pi-t)} .
\end{aligned}
$$

We set $\alpha=1 / 2, \gamma=1 / 3$, and $T=10$. have

Noticing that $\left(H_{1}\right)-\left(H_{2}\right)$ hold, for each $t \in \mathbb{N}_{-1 / 2,21 / 2}$, we

$$
\begin{aligned}
& |p(t, u)-p(t, v)|=\frac{4 t}{15000\left(2+e^{-t}\right)}|u-v|, \\
& |f(t, u)-f(t, v)|=\frac{2+3 t}{(100 \pi-2 t)^{2}}|u-v|, \\
& |F[t, u]-F[t, v]|=\left(\frac{\left(t^{2}+5\right)}{100 \pi-t}\right)\left(\frac{|u|}{10}+\frac{\pi}{8}\right) .
\end{aligned}
$$

Thus,

$$
\begin{aligned}
\|\phi\| & =0.000389 \\
\|\psi\| & =0.00139 \\
\|\omega\| & =0.441
\end{aligned}
$$

Finally, we find that

$$
\begin{aligned}
& \widehat{f}_{0}=0.00389, \\
& \widehat{p}_{0}=0.00105 .
\end{aligned}
$$

So, $\left(H_{3}\right)$ holds with a number $R \in[730.5887,892.9406]$.

Hence, by Theorem 13, problem (59) has a unique solution on $\mathbb{N}_{-1 / 2,21 / 2}$.

Example 2. Consider the following fractional difference boundary value problem:

$$
\begin{aligned}
& \Delta^{1 / 2}\left[\frac{\Delta^{2 / 5} u(t)-\Delta^{-1 / 3} g(t-4 / 15, u(t-4 / 15))}{h(t, u(t))}\right] \\
& =H\left[t-\frac{1}{10}, u\left(t-\frac{1}{10}\right), \Delta^{-3 / 4} u\left(t+\frac{13}{20}\right)\right], \\
& u\left(-\frac{11}{10}\right)=\Delta^{2 / 5} u\left(-\frac{1}{2}\right)=0,
\end{aligned}
$$

for $t \in \mathbb{N}_{0,10}$, where

$$
\begin{aligned}
& g(t, u(t))=\log \left(1+\frac{|u(t)| e^{t} \sin 5 \pi t}{1+|u(t)|}\right), \\
& h(t, u(t))=\left(\frac{|u(t)|+1}{|u(t)|+2}\right) \frac{e^{-\sin ^{2} \pi t}}{100 e-t},
\end{aligned}
$$




$$
\begin{aligned}
H\left[t, u(t), \Delta^{-3 / 4} u\left(t+\frac{3}{4}\right)\right] \\
=\frac{1}{3} \arctan \left(\frac{3|u(t)| \sin ^{2} 5 \pi t}{10+|u(t)|}\right) \\
\quad-\frac{1}{3} \arctan \left(\frac{3\left|\Delta^{-3 / 4} u(t+3 / 4)\right| \sin ^{2} 5 \pi t}{10+\left|\Delta^{-3 / 4} u(t+3 / 4)\right|}\right) .
\end{aligned}
$$

We let $\alpha=1 / 2, \beta=2 / 5, \gamma=1 / 3, \omega=3 / 4$, and $T=10$.

Noticing that $\left(K_{1}\right)-\left(K_{2}\right)$ hold, for each $t \in \mathbb{N}_{-11 / 10,109 / 10}$, we obtain

$$
\begin{aligned}
& |h(t, u)-h(t, v)|=\left(\frac{e^{-\sin ^{2} \pi t}}{400 e-4 t}\right)|u-v|, \\
& \left|H\left[t, u, \Delta^{-3 / 4} u\right]-H\left[t, v, \Delta^{-3 / 4} v\right]\right| \\
& \quad=\frac{1}{10} \sin ^{2} 5 \pi t\left(|u-v|+\left|\Delta^{-3 / 4} u-\Delta^{-3 / 4} v\right|\right), \\
& |h(t, u)|=\frac{e^{-\sin ^{2} \pi t}}{100 e-t}+e^{-\pi t}, \\
& \left|H\left[t, u, \Delta^{-3 / 4} u\right]\right|=3 \sin ^{2} 5 \pi t .
\end{aligned}
$$

Thus, we obtain

$$
\begin{aligned}
\|\vartheta\| & =0.000923, \\
\|\theta\| & =0.1 \\
\|\mu\| & =0.00369 \\
\|\nu\| & =3 .
\end{aligned}
$$

Finally, we find that

$$
\begin{aligned}
& \frac{(T+\alpha+\beta)^{\beta}}{\Gamma(\beta+1)}\left\{\frac{\|\vartheta\|\|\nu\|(T+\alpha+\beta)^{\alpha}}{\Gamma(\alpha+1)}\right. \\
& \left.\quad+\|\mu\|\|\theta\|\left(1+\frac{(T+\alpha+\beta)^{\omega}}{\Gamma(\omega+1)}\right)\right\} \approx 0.2277<1 .
\end{aligned}
$$

Hence, by Theorem 16, problem (64) has a unique solution on $\mathbb{N}_{-11 / 10,109 / 10}$.

\section{Conflicts of Interest}

The authors declare that there are no conflicts of interest regarding the publication of this paper.

\section{Acknowledgments}

This research was funded by King Mongkut's University of Technology North Bangkok, Contract no. KMUTNB-GOV60-71.

\section{References}

[1] G.-C. Wu and D. Baleanu, "Discrete fractional logistic map and its chaos," Nonlinear Dynamics, vol. 75, no. 1-2, pp. 283-287, 2014.

[2] G. C. Wu and D. Baleanu, "Chaos synchronization of the discrete fractional logistic map," Signal Processing, vol. 102, pp. 96-99, 2014.

[3] C. S. Goodrich and A. C. Peterson, Discrete Fractional Calculus, Springer, New York, NY, USA, 2015.

[4] F. M. Atici and P. W. Eloe, "A transform method in discrete fractional calculus," International Journal of Difference Equations, vol. 2, no. 2, pp. 165-176, 2007.

[5] F. M. Atici and P. W. Eloe, "Initial value problems in discrete fractional calculus," Proceedings of the American Mathematical Society, vol. 137, no. 3, pp. 981-989, 2009.

[6] F. M. Atici and P. W. Eloe, “Two-point boundary value problems for finite fractional difference equations," Journal of Difference Equations and Applications, vol. 17, no. 4, pp. 445-456, 2011.

[7] T. Abdeljawad and D. Baleanu, "Fractional differences and integration by parts," Journal of Computational Analysis and Applications, vol. 13, no. 3, pp. 574-582, 2011.

[8] T. Abdeljawad, "On Riemann and Caputo fractional differences," Computers \& Mathematics with Applications, vol. 62, no. 3, pp. 1602-1611, 2011.

[9] T. Abdeljawad and F. M. Atici, "On the definitions of nabla fractional operators," Abstract and Applied Analysis, vol. 2012, Article ID 406757, 2012.

[10] T. Abdeljawad, "Dual identities in fractional difference calculus within Riemann," Advances in Difference Equations, vol. 2013, article no. 36, 2013.

[11] T. Abdeljawad, "On delta and nabla Caputo fractional differences and dual identities," Discrete Dynamics in Nature and Society, vol. 2013, Article ID 406910, 2013.

[12] G. Anastassiou, "Foundations of nabla fractional calculus on time scales and inequalities," Computers \& Mathematics with Applications, vol. 59, no. 12, pp. 3750-3762, 2010.

[13] M. Holm, "Sum and difference compositions in discrete fractional calculus," Cubo, vol. 13, no. 3, pp. 153-184, 2011.

[14] B. Jia, L. Erbe, and A. Peterson, "Two monotonicity results for nabla and delta fractional differences," Archiv der Mathematik, vol. 104, no. 6, pp. 589-597, 2015.

[15] B. Jia, L. Erbe, and A. Peterson, "Convexity for nabla and delta fractional differences," Journal of Difference Equations and Applications, vol. 21, no. 4, pp. 360-373, 2015.

[16] J. Čermák, T. Kisela, and L. Nechvátal, "Stability and asymptotic properties of a linear fractional difference equation," Advances in Difference Equations, vol. 2012, article no. 122, 2012.

[17] F. Jarad, T. Abdeljawad, D. Baleanu, and K. Biçen, "On the stability of some discrete fractional nonautonomous systems," Abstract and Applied Analysis, vol. 2012, Article ID 476581, 2012.

[18] D. Mozyrska and M. Wyrwas, "The Z-transform method and delta type fractional difference operators," Discrete Dynamics in Nature and Society, vol. 2015, Article ID 852734, 2015.

[19] D. Mozyrska and M. g. Wyrwas, "Explicit criteria for stability of fractional $h$-difference two-dimensional systems," International Journal of Dynamics and Control, vol. 5, no. 1, pp. 4-9, 2017.

[20] R. A. C. Ferreira and D. F. M. Torres, "Fractional $h$-difference equations arising from the calculus of variations," Applicable Analysis and Discrete Mathematics, vol. 5, no. 1, pp. 110-121, 2011. 
[21] R. A. C. Ferreira, "Existence and uniqueness of solution to some discrete fractional boundary value problems of order less than one," Journal of Difference Equations and Applications, vol. 19, no. 5, pp. 712-718, 2013.

[22] R. A. C. Ferreira and C. S. Goodrich, "Positive solution for a discrete fractional periodic boundary value problem," Dynamics of Continuous, Discrete \& Impulsive Systems, vol. 19, no. 5, pp. 545-557, 2012.

[23] C. S. Goodrich, "Existence and uniqueness of solutions to a fractional difference equation with nonlocal conditions," Computers \& Mathematics with Applications, vol. 61, no. 2, pp. 191-202, 2011.

[24] C. S. Goodrich, "On a discrete fractional three-point boundary value problem," Journal of Difference Equations and Applications, vol. 18, no. 3, pp. 397-415, 2012.

[25] C. S. Goodrich, "A convexity result for fractional differences," Applied Mathematics Letters, vol. 35, no. 1, pp. 58-62, 2014.

[26] C. S. Goodrich, "The relationship between sequential fractional differences and convexity," Applicable Analysis and Discrete Mathematics, vol. 10, no. 2, pp. 345-365, 2016.

[27] R. Dahal and C. S. Goodrich, "A monotonicity result for discrete fractional difference operators," Archiv der Mathematik, vol. 102, no. 3, pp. 293-299, 2014.

[28] L. Erbe, C. S. Goodrich, B. Jia, and A. Peterson, "Survey of the qualitative properties of fractional difference operators: monotonicity, convexity, and asymptotic behavior of solutions," Advances in Difference Equations, vol. 2016, no. 1, article no. 43, pp. 1-31, 2016.

[29] F. Chen, X. Luo, and Y. Zhou, "Existence results for nonlinear fractional difference equation," Advances in Difference Equations, vol. 2011, Article ID 713201, 2011.

[30] F. Chen and Y. Zhou, "Existence and Ulam stability of solutions for discrete fractional boundary value problem," Discrete Dynamics in Nature and Society, vol. 2013, Article ID 459161, 2013.

[31] Y. Chen and X. Tang, "The difference between a class of discrete fractional and integer order boundary value problems," Communications in Nonlinear Science and Numerical Simulation, vol. 19, no. 12, pp. 4057-4067, 2014.

[32] W. Lv, "Solvability for discrete fractional boundary value problems with a p -laplacian operator," Discrete Dynamics in Nature and Society, vol. 2013, Article ID 679290, 2013.

[33] W. Lv, "Solvability for a discrete fractional three-point boundary value problem at resonance," Abstract and Applied Analysis, vol. 2014, Article ID 601092, 7 pages, 2014.

[34] W. Lv and J. Feng, "Nonlinear discrete fractional mixed type sum-difference equation boundary value problems in Banach spaces," Advances in Difference Equations, vol. 2014, no. 1, article no. 184, 2014.

[35] H. Q. Chen, Y. Q. Cui, and X. L. Zhao, "Multiple solutions to fractional difference boundary value problems," Abstract and Applied Analysis, vol. 2014, Article ID 879380, 6 pages, 2014.

[36] H. Q. Chen, Z. Jin, and S. G. Kang, "Existence of positive solutions for Caputo fractional difference equation," Advances in Difference Equations, vol. 44, pp. 1-12, 2015.

[37] S. G. Kang, Y. Li, and H. Q. Chen, "Positive solutions to boundary value problems of fractional difference equation with nonlocal conditions," Advances in Difference Equations, vol. 2014, no. 1, article no. 7, 2014.

[38] W. Dong, J. Xu, and D. O. Regan, "Solutions for a fractional difference boundary value problem," Advances in Difference Equations, vol. 2013, article no. 319, 2013.
[39] T. Sitthiwirattham, J. Tariboon, and S. K. Ntouyas, "Existence results for fractional difference equations with three-point fractional sum boundary conditions," Discrete Dynamics in Nature and Society, vol. 2013, Article ID 104276, 2013.

[40] T. Sitthiwirattham, J. Tariboon, and S. K. Ntouyas, "Boundary value problems for fractional difference equations with threepoint fractional sum boundary conditions," Advances in Difference Equations, vol. 2013, article no. 296, 2013.

[41] T. Sitthiwirattham, "Existence and uniqueness of solutions of sequential nonlinear fractional difference equations with three-point fractional sum boundary conditions," Mathematical Methods in the Applied Sciences, vol. 38, no. 13, pp. 2809-2815, 2015.

[42] T. Sitthiwirattham, "Boundary value problem for $p$-Laplacian Caputo fractional difference equations with fractional sum boundary conditions," Mathematical Methods in the Applied Sciences, vol. 39, no. 6, pp. 1522-1534, 2016.

[43] S. Chasreechai, C. Kiataramkul, and T. Sitthiwirattham, "On Nonlinear Fractional Sum-Difference Equations via Fractional Sum Boundary Conditions Involving Different Orders," Mathematical Problems in Engineering, vol. 2015, Article ID 519072, 2015.

[44] J. Reunsumrit and T. Sitthiwirattham, "Positive solutions of three-point fractional sum boundary value problem for Caputo fractional difference equations via an argument with a shift," Positivity, vol. 20, no. 4, pp. 861-876, 2016.

[45] J. Reunsumrit and T. Sitthiwirattham, "On positive solutions to fractional sum boundary value problems for nonlinear fractional difference equations," Mathematical Methods in the Applied Sciences, vol. 39, no. 10, pp. 2737-2751, 2016.

[46] J. Soontharanon, N. Jasthitikulchai, and T. Sitthiwirattham, "Nonlocal fractional sum boundary value problems for mixed types of Riemann-Liouville and Caputo fractional difference equations," Dynamic Systems and Applications, vol. 25, no. 3, pp. 409-429, 2016.

[47] S. Laoprasittichok and T. Sitthiwirattham, "Existence and uniqueness results of nonlocal fractional sum-difference boundary value problems for fractional difference equations involving sequential fractional difference operators," Journal of Computational Analysis and Applications, vol. 23, no. 6, pp. 1097-1111, 2017.

[48] B. Kaewwisetkul and T. Sitthiwirattham, "On nonlocal fractional sum-difference boundary value problems for Caputo fractional functional difference equations with delay," Advances in Difference Equations, vol. 2017, no. 1, article no. 219, 2017.

[49] M. Caputo, "Linear models of dissipation whose $Q$ is almost frequency independent-II," The Geophysical Journal of the Royal Astronomical Society, vol. 13, no. 5, pp. 529-539, 1967.

[50] K. Diethelm and N. J. Ford, "Analysis of fractional differential equations," Journal of Mathematical Analysis and Applications, vol. 265, no. 2, pp. 229-248, 2002.

[51] K. Diethelm and N. J. Ford, "Multi-order fractional differential equations and their numerical solution," Applied Mathematics and Computation, vol. 154, no. 3, pp. 621-640, 2004.

[52] A. M. A. El-Sayed and A.-G. Ibrahim, "Set-valued integral equations of fractional-orders," Applied Mathematics and Computation, vol. 118, no. 1, pp. 113-121, 2001.

[53] V. Gafiychuk, B. Datsko, and V. Meleshko, "Mathematical modeling of time fractional reaction-diffusion systems," Journal of Computational and Applied Mathematics, vol. 220, no. 1-2, pp. 215-225, 2008. 
[54] J. H. He, "Approximate analytical solution for seepage flow with fractional derivatives in porous media," Computer Methods Applied Mechanics and Engineering, vol. 167, no. 1-2, pp. 57-68, 1998.

[55] G. Jumarie, "An approach via fractional analysis to non-linearity induced by coarse-graining in space," Nonlinear Analysis: Real World Applications, vol. 11, no. 1, pp. 535-546, 2010.

[56] T. Qiu and Z. H. Bai, "Positive solutions for boundary value problem of nonlinear fractional differential equation," Journal of Nonlinear Sciences and Applications, vol. 2008, pp. 123-131, 2008.

[57] A. K. Alomari, "A novel solution for fractional chaotic Chen system," Journal of Nonlinear Sciences and Applications, vol. 8, no. 5, pp. 478-488, 2015.

[58] Y. Zhao, S. Sun, Z. Han, and Q. Li, "Theory of fractional hybrid differential equations," Computers \& Mathematics with Applications. An International Journal, vol. 62, no. 3, pp. 13121324, 2011.

[59] Y. Zhao and Y. Wang, "Existence of solutions to boundary value problem of a class of nonlinear fractional differential equations," Advances in Difference Equations, vol. 2014, no. 1, 2014.

[60] S. Sun, Y. Zhao, Z. Han, and Y. Li, "The existence of solutions for boundary value problem of fractional hybrid differential equations," Communications in Nonlinear Science and Numerical Simulation, vol. 17, no. 12, pp. 4961-4967, 2012.

[61] B. Ahmad, S. K. Ntouyas, and A. Alsaedi, "Existence results for a system of coupled hybrid fractional differential equations," The Scientific World Journal, vol. 2014, Article ID 426438, 6 pages, 2014.

[62] B. Ahmad and S. K. Ntouyas, "An existence theorem for fractional hybrid differential inclusions of Hadamard type with Dirichlet boundary conditions," Abstract and Applied Analysis, vol. 2014, Article ID 705809, 2014.

[63] B. C. Dhage and S. K. Ntouyas, "Existence results for boundary value problems for fractional hybrid differential inclusions," Topological Methods in Nonlinear Analysis, vol. 44, no. 1, pp. 229-238, 2014.

[64] S. Sitho, S. K. Ntouyas, and J. Tariboon, "Existence results for hybrid fractional integro-differential equations," Boundary Value Problems, vol. 2015, no. 1, article no. 113, pp. 1-13, 2015.

[65] T. Bashiri, S. M. Vaezpour, and C. Park, "Existence results for fractional hybrid differential systems in Banach algebras," Advances in Difference Equations, vol. 2016, no. 1, article no. 57, pp. 1-13, 2016.

[66] D. H. Griffel, Applied Functional Analysis, Ellis Horwood Publishers, Chichester, UK, 1981.

[67] B. C. Dhage, "A fixed point theorem in Banach algebras with applications to fractional integral equations," Kyungpook Mathematical Journal, vol. 44, pp. 145-155, 2004.

[68] B. C. Dhage, "A nonlinear alternative with applications to nonlinear perturbed differential equations," Nonlinear Studies, vol. 13, no. 4, pp. 343-354, 2006. 


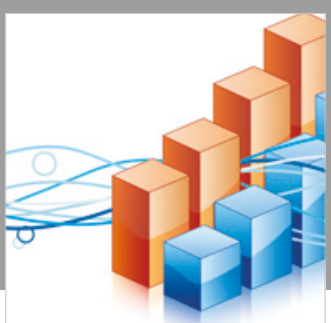

Advances in

Operations Research

\section{-n-m}
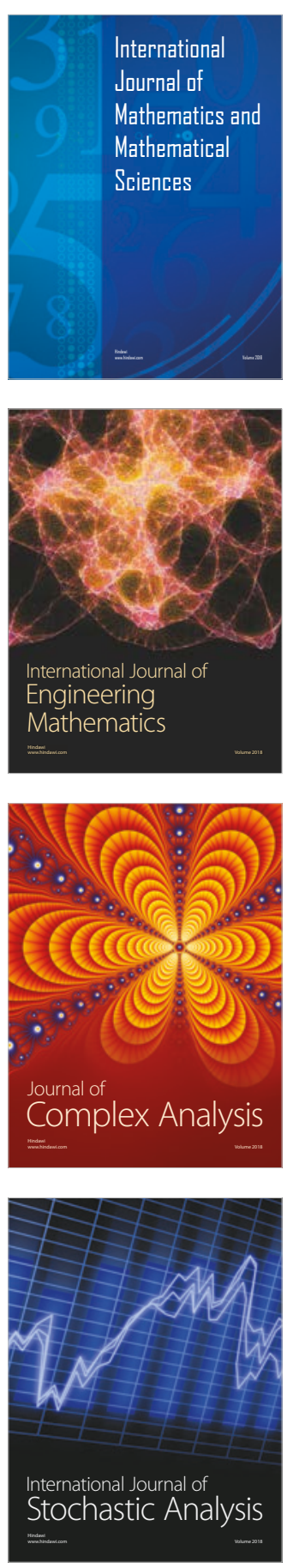
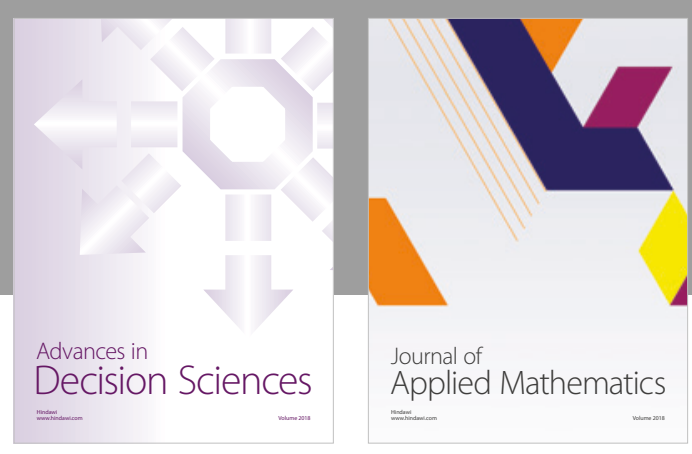

Journal of

Applied Mathematics


Submit your manuscripts at

www.hindawi.com

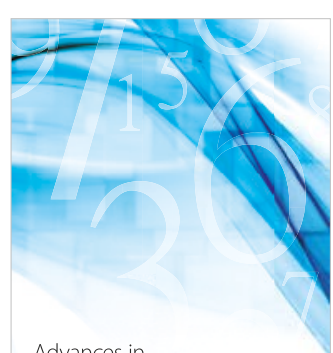

Advances in
Numerical Analysis
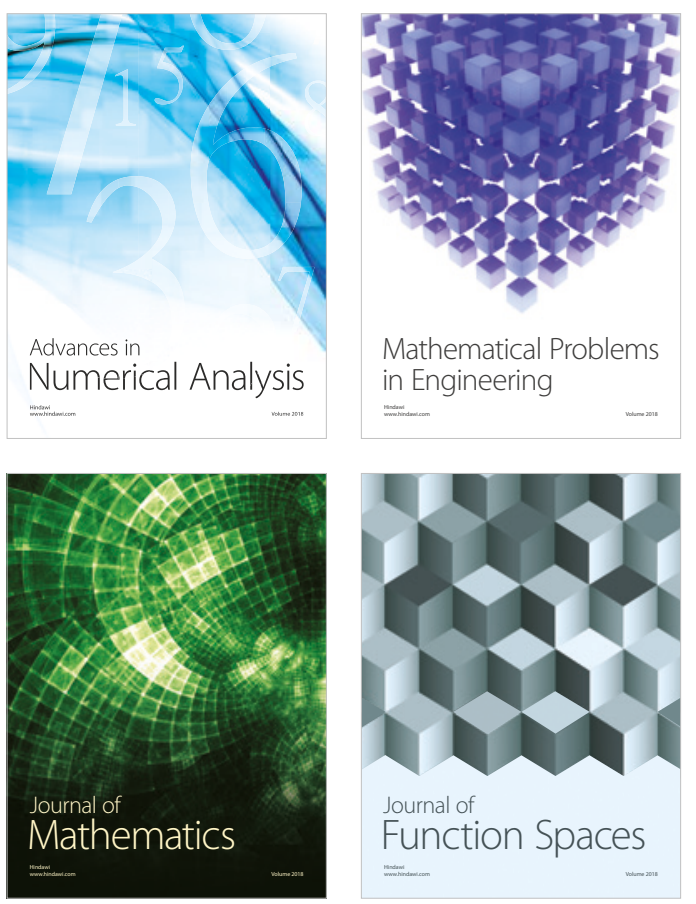

Mathematical Problems in Engineering

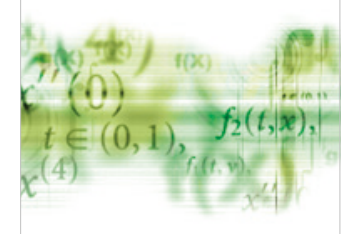

International Journal of

Differential Equations

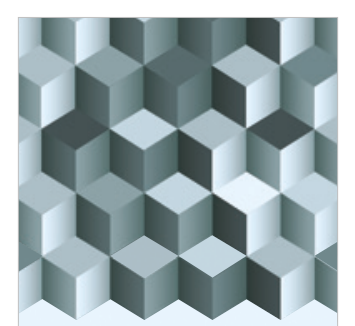

Journal of

Function Spaces



The Scientific

World Journal

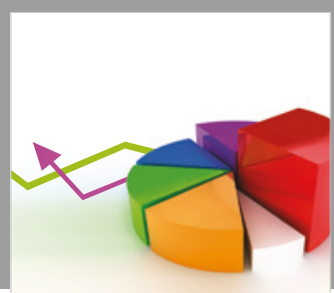

Journal of

Probability and Statistics
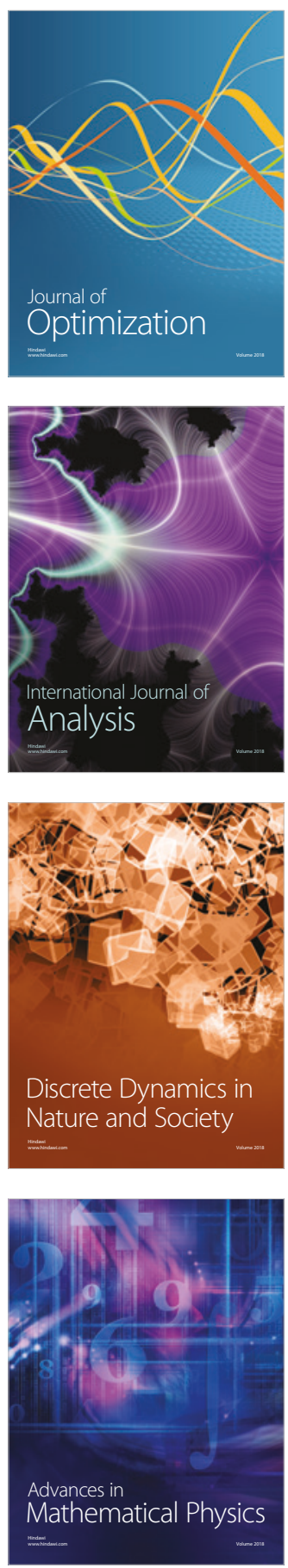